Reprod. Nutr. Dévelop., 1988, 28 (1), 201-202.

\title{
Interférence du soufre et du molybdène avec le métabolisme du cuivre et du zinc chez l'agneau
}

M. LAMAND, C. LAB, M. MIGNON, J. C. TRESSOL

Laboratoire des Maladies Nutritionnelles, I.N.R.A., Theix, 63122 Ceyrat, France.

Summary. A diet containing $5 \mathrm{~g}$ of sulfur $/ \mathrm{kg}$ dry matter and different increasing levels of molybdenum induced a thiomolybdate synthesis in lambs, as detected by a copper TCA insoluble fraction appearing in the blood of these animals. The minimum level of molybdenum to trigger the S-Mo-Cu interference was around $3 \mathrm{mg} \mathrm{Mo} / \mathrm{kg} \mathrm{DM}$.

The sulfur of the diet reduced the zinc availability, as the zincaemia of some animals fell below the deficiency limit.

A un niveau normal, le soufre est utilisé dans la synthèse d'acides aminés soufrés par les microbes du rumen. Les excès de soufre sont fréquents, soit par introduction abusive de soufre dans les mélanges minéraux, soit par contamination d'origine industrielle (addition de sulfates comme adjuvant de surpressage des pulpes).

En présence de molybdène et des sulfures provenant du soufre en excès, des di- et tri-thiomolybdates apparaissent dans le rumen (Mason, 1982). Les thiomolybdates sont des inhibiteurs des enzymes cuprodépendantes. Nous avons voulu savoir à partir de quelle dose de molybdène les thiomolybdates sont synthétisés et à quelle dose l'interférence S-Mo-Cu apparaît. Nous avons détecté et suivi I'apparition des thiomolybdates par le dosage d'une fraction (anormale) de cuivre plasmatique insoluble en milieu trichloracétique $5 \%$ (Lamand et al., 1980).

Matériel et méthodes. Vingt agneaux Limousin $\times$ Romanoff de $35 \mathrm{~kg}$ en moyenne ont été répartis en 4 lots de 5 animaux. Ils ont tous reçu un aliment concentré (maïs $40 \%$, luzerne déshydratée $60 \%$ ) supplémenté avec $3,2 \mathrm{~g}$ de soufre $/ \mathrm{kg}$ (les besoins étant de $2 \mathrm{~g} \mathrm{~S} / \mathrm{kg}$ MS dans l'ensemble de la ration) à raison de $1 \mathrm{~kg} /$ jour et $200 \mathrm{~g}$ de foin. Avec un complément minéral, le régime contenait $7 \mathrm{mg} \mathrm{Cu} / \mathrm{kg} \mathrm{MS}$ et $85 \mathrm{mg} \mathrm{Zn} / \mathrm{kg} \mathrm{MS}$.

Dans les lots 1 à 4 , les teneurs des régimes en Mo ont été respectivement portées à 2,$4 ; 4,8 ; 7,2 ; 9,6 \mathrm{mg} / \mathrm{kg}$ MS par du molybdate d'ammonium distribué per os.

Les oligoéléments et la fraction de cuivre plasmatique insoluble dans l'acide trichloracétique (TCA) à $5 \%$ ont été dosés comme précédemment (Lamand et al., 1980). L'évolution de ce dernier paramètre a été suivie pour chaque lot par le test $U$ non paramétrique de Mann et Whitney et pour l'ensemble des lots par le test $\mathrm{H}$ de Kruskall-Wallis (Snedecor et Cochran. 1957).

Résultats et discussion. Dès le $4^{\mathrm{e}}$ jour et pour l'ensemble des animaux des lots $2,3,4$, la distribution du molybdène a provoqué une augmentation significative de la fraction du cuivre plasmatique insoluble dans le TCA (fig. 1). 
L'interférence du soufre et du molybdène avec le cuivre est donc précoce. En revanche, la dose minimum de Mo (lot 1) n'a pas eu d'effet significatif. La limite de toxicité du molybdène avec une teneur en soufre voisine de $5 \mathrm{~g} / \mathrm{kg}$ MS est donc comprise entre 2,4 et $4,8 \mathrm{mg} \mathrm{Mo} / \mathrm{kg} \mathrm{MS}$. On peut considérer que même avec un apport de soufre relativement modéré, la limite de toxicité du molybdène est proche des $3 \mathrm{mg} \mathrm{Mo} / \mathrm{kg}$ MS suggérés par Underwood (1966).

La fraction de cuivre plasmatique insoluble dans le TCA augmente jusqu'à un plateau situé autour de 2,$4 ; 3,4 ; 5,6 \mu \mathrm{mol} / /$ pour les lots $2,3,4$ à partir du $7 \mathrm{e}$ jour. Lorsque l'équilibre est atteint on peut estimer que cette fraction est proportionnelle à l'apport de molybdène $(r=0,98)$.

Le niveau de molybdène n'ayant pas d'influence sur la zincémie des animaux, ce paramètre a été traité sur toute la population.

Malgré un apport de zinc supérieur aux recommandations $(85 \mathrm{mg} / \mathrm{kg} \mathrm{MS})$, la zincémie descend de $19 \pm 2,9 \mu \mathrm{mol} / \mathrm{l}$ à $11,2 \pm 1,6 \mu \mathrm{mol} / \mathrm{l}$ en 34 jours (fig. 2 ). La limite de carence se situant vers $11 \mu \mathrm{mol} / \mathrm{l}$, environ la moitié des animaux sont hypozincémiques. Un apport de soufre interfère donc avec l'absorption du zinc, ce qui peut entraîner des carences en cet élément à plus ou moins long terme. Ce résultat confirme des résultats antérieurs obtenus par la méthode des bilans (Lamand, 1974). Une telle interférence peut expliquer les carences en zinc (cliniques) observées chez des ruminants recevant une complémentation minérale tout à fait suffisante en zinc mais trop riche en soufre.

On peut donc conclure qu'en présence d'un excès de soufre, le molybdène commence à interférer à partir de $3 \mathrm{mg} \mathrm{Mo} / \mathrm{kg} M S$ et que le soufre, vers $5 \mathrm{~g} / \mathrm{kg}$ MS, peut diminuer la digestibilité du zinc d'un régime normal en cet élément.
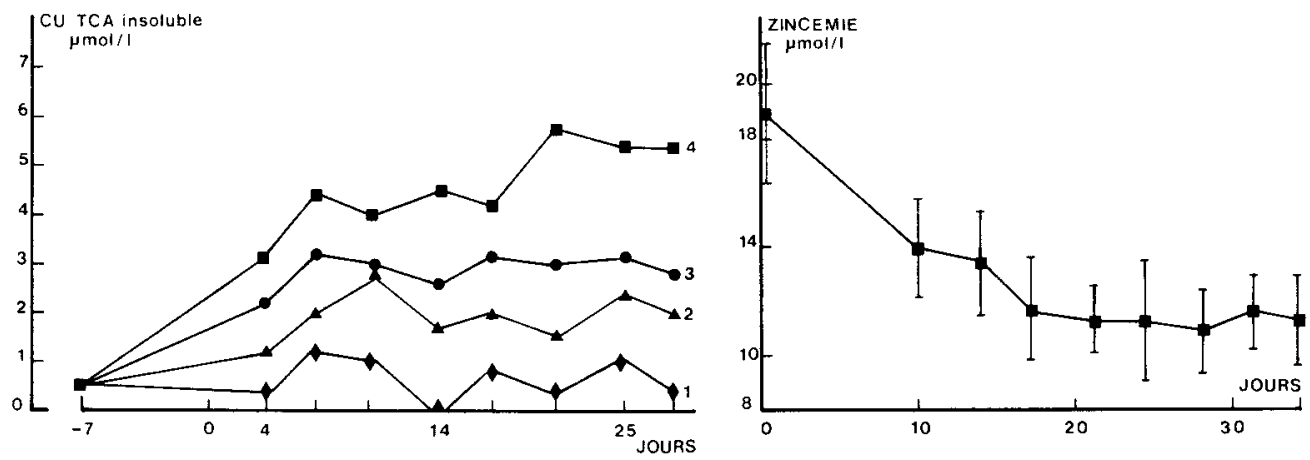

FIG. 1. - Evolution de la fraction de cuivre plasmatique TCA insoluble dans les 4 lots d'agneaux FIG. 2. - Evolution de la zincémie de l'ensemble des animaux. recevant une ration plus ou moins riche en molybdène. Jour $\mathrm{O}=$ début de la distribution du molybdène. $1: 2,4 \mathrm{mg} \mathrm{Mo} / \mathrm{kg} \mathrm{MS} ; 2: 4,8 \mathrm{mg}$ $\mathrm{Mo} / \mathrm{kg} \mathrm{MS} ; 3: 7,2 \mathrm{mg} \mathrm{Mo} / \mathrm{kg} \mathrm{MS} ; 4: 9,6 \mathrm{mg}$ $\mathrm{Mo} / \mathrm{kg}$ MS.

Lamand M., 1974. Ann. Rech. vét., 5, 281-289.

Lamand M., Lab C., Tressol J. C., Mason J., 1980. Ann. Rech. vét., 11, 141-145.

Mason J., 1982. Irish vet. J., 36, 164-168.

Snedecor G. W., Cochran W. G., 1957. Statistical methods. The lowa State Univ. Press Ames lowa, USA, p. 649 .

Underwood E. J., 1966. The mineral nutrition of livestock. FAO, Commonwealth Agric. Bureau ed. 